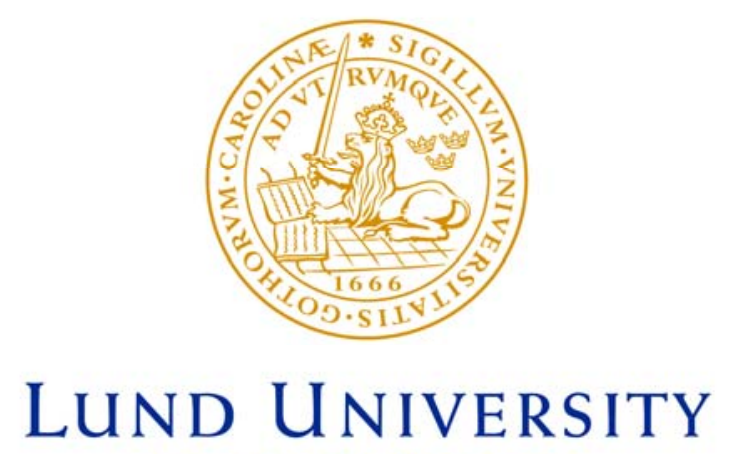

Faculty of Medicine

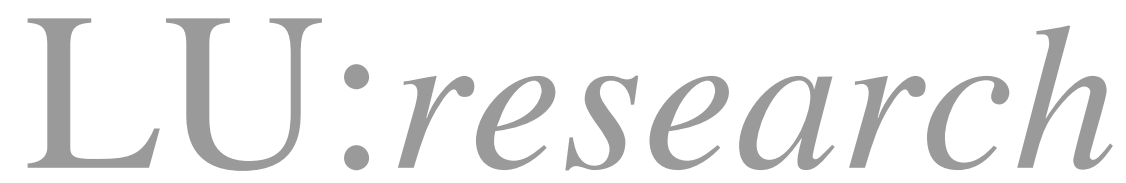

Institutional Repository of Lund University

This is an author produced version of a paper published in

Molecular microbiology. This paper has been peerreviewed but does not include the final publisher proofcorrections or journal pagination.

Citation for the published paper:

Shannon, Oonagh and Hertzén, Erika and Norrby-Teglund, Anna and Morgelin, Matthias and Sjobring, Ulf and Bjorck, Lars.

"Severe streptococcal infection is associated with $M$ protein-induced platelet activation and thrombus formation."

Mol Microbiol, 2007, Vol: 65, Issue: 5, pp. 1147-57.

http://dx.doi.org/10.1111/j.1365-2958.2007.05841.x

Access to the published version may require journal subscription.

Published with permission from: Blackwell 


\title{
Severe streptococcal infection is associated with $M$ protein-induced platelet activation and thrombus formation
}

\author{
Oonagh Shannon ${ }^{1}$, Erika Hertzén ${ }^{2}$, Anna Norrby-Teglund ${ }^{2}$, Matthias \\ Mörgelin ${ }^{1}$, Ulf Sjöbring ${ }^{1,3}$ and Lars Björck ${ }^{1}$
}

1. From the Department of Clinical Sciences, Section of Infection Medicine, Lund University, Lund, Sweden.

2. Center for Infectious Medicine, Department of Medicine, Karolinska Institutet, Karolinska University Hospital, Huddinge, Sweden.

3. Department of Laboratory Medicine, Lund University, Lund, Sweden.

Reprints: Oonagh Shannon, Department of Clinical Sciences, Biomedical Centre (BMC), B14, Lund University, SE- 22184 Lund, Sweden. Email:

oonagh.shannon@med.lu.se. Telephone: +46 46 2220357. Fax: +46 46157756.

Running Title: Platelet activation in streptococcal infection

Research grants: This work was supported by the Swedish Research Council (projects 7480 and 12610), the Österlund foundations and the foundation of Greta and Johan Kocks, Hansa Medical AB, and EC project “AMIS” (contract number LSHM-CT2004-S12093)

Scientific heading: Pathogenicity and virulence 


\section{Summary}

Disturbed hemostasis is a central finding in severe $S$. pyogenes infection. In particular, microthrombi are found both at the local site of infection and at distant sites. Platelets are responsible for maintaining vascular function and hemostasis. We report here that M1 protein of $S$. pyogenes triggers immune mediated platelet activation and thrombus formation. M1 protein is released from the bacterial surface and forms complexes with plasma fibrinogen. These complexes bind to the fibrinogen receptor on resting platelets. When these complexes also contain IgG against M1 protein, this will engage the $\mathrm{Fc}$ receptor on the platelets and activation will occur. Activation of the platelets leads to platelet aggregation and the generation of platelet rich thrombi. Neutrophils and monocytes are in turn activated by the platelets. Platelet thrombi are deposited in the microvasculature and aggregated platelets, IgG and M1 protein co-localise in biopsies from patients diagnosed with $S$. pyogenes toxic shock syndrome. This chain of events results in a pro-coagulant and pro-inflammatory state typical of severe $S$. pyogenes infection. 


\section{Introduction}

Streptococcus pyogenes is a significant human specific pathogen. S. pyogenes frequently causes mild infection, however serious or life threatening infections such as invasive disease, necrotizing fasciitis and sepsis also occur (Cunningham, 2000). In fact, it was recently estimated that $S$. pyogenes causes $>500,000$ deaths/year globally (Carapetis et al., 2005). Sepsis and invasive S. pyogenes infection are often associated with coagulopathy, with a massive dysregulation of the pro- and anti-coagulation equilibrium (Stevens, 2001). This results in deposition of microthrombi in the microvasculature and a consumption of coagulation factors and platelets, with a secondary risk for bleeding. Thrombocytopenia is a central finding in S. pyogenes infections and the severity of sepsis correlates with the decrease in platelet count (Mavrommatis et al., 2000). The interactions between S. pyogenes and the cellular components of coagulation have to date been poorly studied.

Platelets are central players in hemostasis. Resting platelets patrol the vasculature and become activated in response to tissue damage. Adhesion and activation results in the release of the contents of platelet granulae and in the upregulation of surface receptors for fibrinogen $(\mathrm{Fg})$ and other plasma proteins. This allows firm binding between platelets to occur, resulting in aggregation and formation of platelet thrombi. Platelets also have a role in the innate immune response and inflammation, by release of procoagulant factors, growth factors, and pro-inflammatory factors including cytokines, chemokines, and complement proteins (Weyrich et al., 2003). Platelets also adhere to and modulate the function of endothelial cells and leucocytes, and are key players at the crossroads between hemostasis and inflammation. Platelets respond to important pro-inflammatory mediators, which are released in response to microbial challenge (Zimmerman et al., 2002) but can also be directly stimulated by microbial agents, such as endotoxin from Gram negative bacteria (Wachowicz et al., 1998) (Andonegui et al., 2005) (Stahl et al., 2006). A number of significant Gram positive pathogens, including various species of streptococci and Staphylococcus aureus, have also been shown to stimulate platelet activation in vitro (Fitzgerald et al., 2006a). It has previously been shown that $S$. pyogenes bacteria can adhere to and aggregate platelets (Sjöbring et al., 2002). In this study we set about to further elucidate the interactions between $S$. pyogenes and platelets. 
An important virulence factor of $S$. pyogenes is the cell surface-associated M protein (Fischetti, 1989). Based on sequence variations in the $M$ protein, more than one hundred serotypes of $S$. pyogenes have been identified. S. pyogenes strains expressing the M1 protein are the most frequently isolated strains from patients with severe invasive $S$. pyogenes infections. The M1 protein binds specifically and with high affinity to several plasma proteins, including Fg. Importantly, M1 protein can be released from the bacterial surface by three distinct mechanisms; shedding during growth (Åkesson et al., 1994), cleavage by a S. pyogenes cysteine protease (SpeB) (Berge and Björck, 1995) or cleavage by neutrophil proteinases (Herwald et al., 2004). Soluble M1 protein retains functional activity and is a potent activator of neutrophils (Herwald et al., 2004) and monocytes (Påhlman et al., 2006). In the present study we show that soluble M1 protein is a powerful platelet agonist. M1 protein forms complexes with plasma $\mathrm{Fg}$, which engage the low affinity GPIIb/IIIa receptor on platelets. This in itself is not sufficient for platelet activation, which also requires the binding of M1-specific immunoglobulin $\mathrm{G}$ ( $\mathrm{IgG}$ ) to the platelet $\mathrm{Fc}$ gamma receptor II. Activation of platelets by M1 results in the formation of homotypic platelet thrombi as well as heterotypic platelet-leucocyte aggregates, resulting in activation of all cell types. We demonstrate the presence of platelet rich thrombi in the microcirculation of patients suffering from severe $S$. pyogenes infection. Strikingly, the platelet thrombi co-localize with M1 protein and IgG, demonstrating that platelet activation by M1 protein occurs in severe invasive $S$. pyogenes infection. 


\section{Results}

\section{Platelets are activated during $S$. pyogenes infection}

In severe $S$. pyogenes infection microthrombi are formed, which lodge in the microvasculature of diverse tissues. We hypothesized that activated platelets contribute to these microthrombi. Immunohistochemistry of biopsies from patients suffering from soft tissue infections caused by S. pyogenes, proved this to be the case. Platelets could be demonstrated in the tissue through staining for CD42a, and they appeared as large aggregates, consistent with microthrombi formation (Figure 1). We set about to identify the bacterial product that mediates this potent platelet activation and thrombus formation.

We determined platelet activation using three-colour flow cytometry following stimulation of platelets in PRP with M1 bacteria (AP1) or proteins purified from this strain. Platelets were identified using the antibody CD42PerCP, the activation status of GPIIb/IIIa was determined using an antibody specific for the active conformation (PAC-1.FITC), and mobilisation of the alpha granules was determined using the antibody CD62PE. Background numbers of resting platelets were positive for CD62 or PAC-1, and as expected the number of platelets expressing these markers was rapidly increased in response to ADP (Table 1). S. pyogenes bacteria $\left(1 \times 10^{8} / \mathrm{ml}\right)$ of the AP1 strain stimulated platelet activation (Table 1). Purified M1 protein (1 $\mu \mathrm{g} / \mathrm{ml})$ was also a powerful platelet activator, generating activation at levels equivalent to those mediated by ADP and the whole bacteria (Table 1). In contrast, soluble protein $\mathrm{H}$ failed to activate platelets (Table 1). Protein $\mathrm{H}$ is an M-like protein produced by $S$. pyogenes AP1, which unlike M1 protein has no affinity for Fg. In order to localise the region of the M1 protein that mediates platelet activation, fragments A-S and S-C3 of M1 protein were tested. M1 fragment A-S (M1.A-S) contains amino acid 42 to 232 of M1, including the Fg binding B repeats. M1 fragment S-C3 (M1.S-C3) contains amino acids 196-358 of M1 and does not bind to Fg (Åkesson et al., 1994). M1.A-S activated platelets in plasma to an equivalent level as the whole protein, while M1.SC3 failed to activate platelets (Table 1). The $\mathrm{N}$ terminal region of $\mathrm{M} 1$ protein, containing the Fg binding region is therefore responsible for platelet activation. 
In order to determine the role of Fg and other possible plasma cofactors for platelet activation by the soluble M1 protein, we carried out assays in the presence of specific blockers; ReoPro blocks Fg-binding and AT10 blocks IgG-binding to platelets, whereas IdeS degrades plasma IgG. In a pilot experiment $50 \mu \mathrm{g} / \mathrm{ml}$ of ReoPro was determined to be 5 times the concentration required to abolish Fg binding (data not shown). Pretreatment of platelets with ReoPro abolished PAC-1 binding by ADP activated platelets, indicating that the integrin receptor was completely blocked at this concentration of ReoPro (Figure 2). Platelet activation in response to ADP was not affected by AT10 or IdeS. This is to be expected since IgG binding to platelets via Fc gamma RIIA is not involved in the activation of platelets by ADP. Activation of platelets by M1 protein was significantly diminished by preincubation of PRP with ReoPro, AT10, or IdeS (Figure 2), indicating that both plasma Fg and IgG are required in order for M1 to activate platelets.

\section{M1 protein binds to and activates platelets}

FITC labelled M1 was incubated with platelets in plasma the platelets were co-stained with a marker of platelet activation (CD62PE). M1.FITC bound to resting platelets and mediated activation (Figure 3A). Both binding of M1 protein to platelets and activation of platelets by M1 protein were significantly decreased in the presence of ReoPro (Figure 3A). This demonstrates an essential role for $\mathrm{Fg}$ in mediating both binding to and subsequent activation of platelets by M1 protein. In contrast, pretreatment of platelets with AT10 did not affect M1 protein binding but significantly decreased activation of platelets by M1 protein (Figure 3A). This indicates that platelet Fc gamma RIIA is not involved in the binding of M1 protein, but is essential for the activation of platelets by M1 protein.

Under physiological conditions, plasma Fg binds very poorly to resting platelets. M1 protein has two binding sites for Fg (Åkesson et al., 1994) and in plasma M1 protein forms complexes with Fg at the M1 protein concentrations used here (Herwald et al., 2004). Our results suggested that Fg in these complexes interact with the low affinity form of GPIIb/IIIa on resting platelets. In order to test this hypothesis we treated PRP with a cocktail of inhibitors that prevent the activation of platelets. The activation status of GPIIb/IIIa was monitored using a monoclonal antibody (PAC-1), and no 
activation could occur in the presence of the cocktail of inhibitors, even after treatment with the platelet agonist, ADP (Figure 3B). M1 protein was capable of binding to these inactivated platelets even in the presence of the cocktail of inhibitors (Figure 3B). This demonstrates that the M1-Fg complexes bind to the inactive conformation of GPIIb/IIIa.

\section{M1 protein-specific IgG is essential to mediate platelet activation by M1 protein}

\section{in human plasma}

In order to determine whether the activating $\operatorname{IgG}$ antibodies were specific for M1 protein, we determined the IgG levels against M1 protein in serum samples from 5 different donors and a control sample of purified IgG against M1 protein (Figure 4A). There was considerable variation in M1 protein titres among donors and one donor had no antibodies against M1 protein (Figure 4A). The ability of M1 protein to activate the platelets of the same five donors was also determined (Figure 4B). In PRP from the donor lacking IgG against M1 protein, platelets failed to become activated in response to M1 protein (closed triangles, Figure 4B). When the PRP from this nonresponsive donor was supplemented with purified $\operatorname{IgG}$ against M1 protein, platelet activation in response to M1 protein could occur (open triangles, Figure 4B). FITC labelled M1 protein was capable of binding to the platelets of the non-responsive donor (data not shown). Taken together with the previous data, this indicates that specific IgG binds to the M1-Fg complexes and when this IgG interacts with the Fc receptor on platelets, activation occurs.

\section{Activated platelets adhere to and activate neutrophils and monocytes.}

Activated platelets have previously been reported to bind to and co-aggregate leucocytes, particularly neutrophils and monocytes, resulting in activation of the leucocytes (de Gaetano et al., 1999) (Li et al., 2000). We found that whole blood incubated with M1 protein $(1 \mu \mathrm{g} / \mathrm{ml})$ contained a large proportion of platelet-monocyte and platelet-neutrophil aggregates (Figure 5). M1 protein has previously been reported to have direct effects on both monocytes and neutrophils, resulting in the activation of both cell types (Herwald et al., 2004) (Påhlman et al., 2006). In order to assess the contribution of M1 protein-mediated platelet activation to aggregate formation, we used platelet activation blockers. As shown in Figure 2, ReoPro and 
AT10 blocked activation of platelets in PRP by M1 protein, and in whole blood ReoPro and AT10 significantly reduced the platelet-monocyte and platelet-neutrophil aggregate formation (Figure 5). These results show that M1 protein induces the formation of platelet-leucocyte aggregates through activation of platelets.

We determined the activation status of the monocytes and neutrophils in complex with platelets by probing them with cell surface markers of activation. Neutrophils in complex with platelets were activated, as shown by surface presentation of CD11b and CD66b on activated cells (Table 2). Monocytes in complex with platelets were also activated, as shown by surface presentation of CD11b and tissue factor on activated cells (Table 2). These results demonstrate that M1 protein activates platelets, and that these activated platelets adhere to and activate monocytes and neutrophils.

\section{M1 stimulates platelet aggregation and thrombus formation}

In response to physiological stimuli, such as ADP, activated platelets rapidly undergo a shape change, develop pseudopodia and adhere to one another to form aggregates. We used scanning electron microscopy to examine platelet adhesion and aggregation in PRP treated with M1 protein. In the absence of activation, platelets existed as mainly single cells (Figure 6A). However, one minute after addition of ADP the platelets appeared activated and had formed aggregates (Figure 6B). Platelets treated with M1 protein $(1 \mu \mathrm{g} / \mathrm{ml})$ for one minute became activated, and large aggregates were generated (Figure 6C). The platelet thrombi formed in response to M1 protein were morphologically similar to those formed in response to ADP. However, the M1 thrombi were larger and more cell dense, showing that platelet activation by M1 protein results in the generation of large platelet rich thrombi.

Platelets, soluble M1 protein, and immunoglobulins are co-localised in $S$. pyogenes infected human tissue

To investigate whether platelet activation and aggregation mediated by M1 protein occurs in vivo during acute infection, we analysed tissue biopsies collected from patients with soft tissue infections caused by M1 S. pyogenes isolates. All biopsies stained positive for $S$. pyogenes, the M1 protein, and showed a heavy infiltration of neutrophils (brown areas, Figure 7). Platelets could be identified in the tissue through 
staining for CD42a, and they occurred in aggregates (brown areas, Figure 7A). Furthermore, the platelet aggregates were localized to areas that also contained bacteria and the M1 protein. To confirm this co-localization, we used confocal microscopy that revealed that the platelet aggregates co-localized with $S$. pyogenes (Figure 7B). Moreover, soluble M1 protein and IgG were also found in the platelet aggregates (Figure 7C). The results demonstrate that M1 protein mediates platelet aggregation in $S$. pyogenes infected tissue, both at the bacterial surface and when released from the surface.

\section{Discussion}

Coagulopathy is a central finding in severe infections caused by $S$. pyogenes and in invasive soft tissue infection and sepsis the induction of microthrombi results in hypoxia and tissue destruction (Stevens, 2001). The molecular mechanisms described here may represent a central event in the generation of these thrombi. M1 protein released from the bacterial surface binds Fg in plasma, and complexes are formed that are capable of engaging the inactive form of the Fg receptor, GPIIb/IIIa, on platelets. Activation will not occur unless there is also a second component present in plasma, namely specific IgG antibodies against M1 protein. These antibodies will also be part of the M1 protein-Fg complexes, where their Fc regions are exposed and recognised by the platelet $\mathrm{IgG}$ receptor $(\mathrm{Fc} \gamma \mathrm{RII})$. The simultaneous interaction with $\mathrm{Fg}$ and $\mathrm{Fc}$ receptors and subsequent intercellular signalling, results in platelet activation. The formation of M1-Fg complexes occurs when the concentration of both proteins reaches an equilibrium (Kantor, 1965) and M1 $(1 \mu \mathrm{g} / \mathrm{ml})$ in the presence of a plasma concentration of fibrinogen is optimal for complex formation (Herwald et al., 2004). At higher or lower M1 concentrations the complex will not occur and this explains why higher concentrations of M1 fail to further increase platelet activation in our experimental setting.

Platelets activated by M1 protein go on to release their pro-inflammatory granule contents and form complexes with neutrophils and monocytes, resulting in the activation of both cell types (Table 2). A crucial element in the genesis of coagulopathy in sepsis is the generation of tissue factor (Levi et al., 2003). We show 
here that activation of platelets by M1 protein, leads to activation of monocytes, redistribution of tissue factor to the surface, thereby creating a procoagulant state. In sepsis the cardinal symptoms are due to a massive and systemic inflammatory response. Activated neutrophils and monocytes are an important source of inflammatory mediators and platelet-leucocyte aggregate formation and activation of both cell types has been reported to correlate with the severity of bacterial sepsis (Russwurm et al., 2002). In the case of Gram positive pathogens, the mediators of this activation are poorly defined. Notably, it has previously been reported that Streptolysin O, secreted by $S$. pyogenes, stimulates the generation of leucoctyeplatelet aggregates in invasive streptococcal infection (Bryant et al., 2005). Our study shows that platelet activation by M1 protein may play an important role in the pro inflammatory response in S. pyogenes sepsis.

In this study, we used tissue biopsies collected from the epicentre of infection in patients with severe soft tissue infections caused by M1 isolates to facilitate direct assessment of platelet activation at the site of infection. The results revealed aggregated platelets that co-localized with $S$. pyogenes and M1 protein, supporting the hypothesis that M1 protein-induced platelet activation occurs in vivo. Furthermore, areas with platelet aggregation also contained neutrophils consistent with the platelet leucocyte interaction found in vitro. Our findings represent, to our knowledge, the first report of activated platelets localised at the site of bacterial infection. The study also demonstrates that soluble M1 protein, IgG, and platelets are co-localised in the infected tissue. Hence, the in vivo data are in complete agreement with the in vitro findings of this study, and underscore a likely role for platelet activation in the pathogenesis of severe invasive $S$. pyogenes infections.

The role of M1 specific IgG in the platelet activation reported here is in agreement with previously reported mechanisms of platelet activation for other Gram positive bacteria, where a requirement for bacterial specific IgG has also been described (Ford et al., 1997) (Sjöbring et al., 2002) (Loughman et al., 2005) (Fitzgerald et al., 2006b). Regarding M1 protein-specific IgG, it is well established that the presence of these antibodies can protect the host from infection (Fischetti, 1989) (Cunningham, 2000). However, the implications of our findings are that these antibodies may contribute to 
the pathogenesis of infection. S. pyogenes infection is extremely complex and the bacteria can cause diverse infections, therefore the role of these antibodies likely depends on the type of infection, the stage of the infectious process, and host factors. M1 protein mediates a powerful activation of neutrophils, resulting in vascular leakage that contributes to the pathogenesis of infection (Herwald et al., 2004). In relation to the present study, it is interesting that M1 protein specific IgG antibodies have recently been shown to be essential for this neutrophil activation (Kahn et al, manuscript in preparation), which further underlies the potentially damaging effects of antibodies against M1 protein in specific manifestations of S. pyogenes infection.

Under normal circumstances, the low affinity Fg receptor GPIIb/IIIa on the platelet surface can only bind immobilised Fg. Platelet activation results in a conformational change in GPIIb/IIIa, which facilitates recognition of soluble Fg. In this study we show that the M1 protein-Fg complex is capable of engaging the low affinity form of the platelet Fg receptor (Figure 3). This is important since under these circumstances, platelet activation can occur in the circulation and will not necessarily be localised to a damaged endothelium, as is normally the case. These thrombi will be carried with the blood and can become lodged throughout the microvasculature in diverse tissue, also at a distant site from the focus of infection. In fact this is often seen in $S$. pyogenes infection, where thrombi occupy diverse tissue locations (Barker et al., 1987) (Ashbaugh et al., 1998). It is also important to highlight the self-perpetuating nature of these interactions. An activated platelet is full of potent platelet activators, which are released on activation and can target resting cells in the vicinity. The reaction will quickly become self-perpetuating resulting in massive coagulation and consumption of platelets and coagulation factors. This is exactly what is seen in severe cases of sepsis (Gawaz et al., 1997) (Kirschenbaum et al., 2002). The severity of the coagulopathy seems to correlate with the severity of the disease, in particular low platelet count is predictive of a poor outcome in sepsis patients.

This study provides the first clear evidence for a direct role of platelet activation in the pathogenesis of $S$. pyogenes infection. As such this may pave the way for the development of novel anti-infectives based on targeting these interactions. Platelets may have a previously unappreciated central role in the generation of the pro- 
inflammatory response in $S$. pyogenes infection, and therefore blocking platelet activation may represent a good adjunct to the treatment of severe S. pyogenes infection.

\section{Experimental procedures}

\section{Bacteria and bacterial proteins}

Streptococcus pyogenes AP1 (S. pyogenes strain 40/58 from the WHO Collaborating Centre for references and research on Streptococci, Institute of Hygiene and Epidemiology, Prague, Czech Republic) was grown in Todd Hewitt broth at $37^{\circ} \mathrm{C}$ in the presence of $5 \% \mathrm{CO}_{2}$. M1 protein was purified from the supernatant of $\mathrm{MC} 25$, an isogenic mutant of AP1, which lacks the membrane-spanning region and therefore secretes M1 into the supernatant (Collin and Olsen, 2000). This has previously been shown to be a highly pure form of M1 protein, lacking detectable contamination with lipoteichoic acid or peptidoglycans (Påhlman et al., 2006). Recombinant M1 protein fragments, fragment A-S and fragment S-C3, were expressed in E. coli and purified as previously described ((Åkesson et al., 1994). Protein $\mathrm{H}$ was purified from Streptococcus pyogenes AP1 (Åkesson et al., 1990). IdeS specifically cleaves IgG to non-functional fragments and was purified as previously described (von PawelRammingen et al., 2002). Protein L is a surface protein from Finegoldia magna that binds the light chains of IgG (Björck, 1988) .

\section{Platelet preparation}

Blood samples were collected from healthy donors who had not taken antiplatelet medication in the previous ten days. Five $\mathrm{ml}$ of blood was collected into citrated vacuum tubes. Centrifugation at $140 \mathrm{~g}$ for 10 minutes produced an upper platelet rich plasma (PRP), which was removed. For some experiments, biochemical inactivation of platelets was achieved by adding prostaglandin E1 $(1 \mu \mathrm{M})$, apyrase $(1 \mathrm{U} / \mathrm{ml})$ and salicylic acid $(80 \mu \mathrm{M})$ to PRP.

\section{Platelet analyses using flow cytometry}

Ten $\mu l$ of PRP was incubated for 5 minutes at room temperature with $50 \mu l$ of HEPES buffer $\mathrm{pH} 7.4$, either in the presence or absence of $1 \mu \mathrm{M}$ adenosine diphosphate 
(ADP) for activation. Five $\mu$ l flourochrome conjugated antibody (CD42 PerCP, CD62 PE, PAC-1 FITC) or isotype control (all from BD Biosciences) was then added and after 20 minutes the incubation was stopped by addition of $0.5 \%$ formaldehyde in ice cold PBS. Incubations were carried out in the presence of washed S. pyogenes AP1 (1 x $10^{8}$ bacteria/ml $)$, M1 protein $(1 \mu \mathrm{g} / \mathrm{ml})$ or protein $\mathrm{H}(10 \mu \mathrm{g} / \mathrm{ml})$. Samples were analysed using a FACSCalibur flow cytometer in logarithmic mode with a gate setting for the CD42 positive platelet population. 50,000 cells were acquired and analysed using Cell Quest software (Becton Dickinson).

In order to assess the role of plasma factors in platelet activation, PRP was treated with specific blockers, ReoPro $(50 \mu \mathrm{g} / \mathrm{ml})$ or AT10 $(50 \mu \mathrm{g} / \mathrm{ml})$, for 30 minutes at room temperature. ReoPro (Eli Lily) is the Fab fragment of a human-murine chimeric monoclonal antibody that binds to GPIIb/IIIa on platelets and abolishes Fg binding to platelets (Coller, 1995). AT10 (Serotec) is a monoclonal antibody that recognises the low affinity IgG receptor (FcyRIIA;CD32) on platelets and blocks Fc mediated binding of IgG to platelets (Greenman et al., 1991). IdeS is a streptococcal protein that specifically cleaves IgG to non-functional fragments (von Pawel-Rammingen et al., 2002). PRP was treated with $40 \mu \mathrm{g} / \mathrm{ml} \mathrm{IdeS}$ at $37^{\circ} \mathrm{C}$ in order to inactivate the IgG in plasma.

\section{M1 protein binding to platelets}

M1 was FITC conjugated with an EZ-label FITC protein labelling kit (Pierce Biotechnology, Rockford, Illinois) according to the manufacturers instructions. Platelets in PRP were incubated with M1 FITC at various concentrations for 5 minutes at room temperature. Five $\mu 1$ of flourochrome-conjugated antibody (CD42 PerCP, CD62 PE) or isotype control was then added, and after 20 minutes the incubation was stopped on addition of $0.5 \%$ formaldehyde in ice cold PBS. Samples were analysed using a FACSCalibur flow cytometer.

\section{Anti-M1 protein antibody levels in plasma}

Serum was prepared from clotted blood samples by centrifugation at $2000 \mathrm{~g}$ for 10 minutes. A control sample of M1 protein-specific IgG was prepared from a pool of human IgG purified from healthy individuals (Gamimune N 10\%, Bayer), using M1 
protein-coupled sepharose. Microtiter wells were coated with $200 \mu \mathrm{l}$ of Fg $(25 \mu \mathrm{g} / \mathrm{ml})$ overnight at $4^{\circ} \mathrm{C}$. The wells were washed three times with PBS containing $0.05 \%$ Tween (PBST). M1 protein $(1.25 \mu \mathrm{g} / \mathrm{ml})$ was added to each well and incubated at $37^{\circ} \mathrm{C}$ for one hour. After washing with PBST the wells were blocked with $2 \%$ Bovine serum albumin (BSA) in PBST and incubated at $37^{\circ} \mathrm{C}$ for 30 minutes. After washing with PBST, the prepared donor sera or M1 protein specific IgG was added. After incubation for one hour at $37^{\circ} \mathrm{C}$ and washing with PBST, HRP labelled protein $\mathrm{G}$ was added to each well and incubated for one hour at $37^{\circ} \mathrm{C}$. The wells were washed and the reaction was developed using ABTS development fluid. The absorbance was read at $415 \mathrm{~nm}$.

\section{Flow cytometry of platelet-leucocyte aggregates}

The formation of platelet-leucocyte aggregates in the presence of M1 protein was determined using three colour flow cytometry (Li et al., 1997). One hundred $\mu 1$ of citrated whole blood was incubated with ADP $(5 \mu \mathrm{M})$ or M1 protein $(1 \mu \mathrm{g} / \mathrm{ml})$. Ten $\mu 1$ of flourochrome conjugated antibody (CD42 PerCP (BD Biosciences), CD45 FITC or CD45 PE (DAKO Cytomation)) or isotype control was then added. After 20 minutes at RT, the red blood cells were lysed using the Uti.Lyse kit from DAKO Cytomation. Samples were analysed using a FACSCalibur flow cytometer with a gate setting for the CD45 positive leucocyte population. The monocyte and neutrophil populations were identified by their characteristic size and granularity.

The activation status of the leucocytes in complex with platelets was determined by including monoclonal antibodies against activation dependent cell surface markers; CD11bPE, FITC labelled CD66b (BD Biosciences) or FITC labelled anti-tissue factor (Abcam).

\section{Scanning electron microscopy of platelets}

Platelet morphology in the presence of M1 protein was monitored using scanning electron microscopy. Two hundred $\mu$ l of PRP was incubated with PBS, ADP (5 $\mu \mathrm{M})$ or M1 $(1 \mu \mathrm{g} / \mathrm{ml})$ for 1 minute before addition of $1 \mathrm{ml}$ of fixation fluid $(2.5 \%$ glutaraldehyde in $0.15 \mathrm{M}$ sodium cacodylate, $\mathrm{pH} 7.4$ ). After one hour at RT, the cells were sedimented at $1000 \mathrm{~g}$ for 10 minutes and absorbed onto poly-1-lysine coated 
coverslips. After 1 hour, the samples were immersed in fixation fluid and incubated overnight. The samples were washed with cacodylate buffer, dehydrated, critical point dried and sputtered with 30nm gold. The samples were analysed using a Jeol J-330 scanning electron microscope and images were recorded with a Gatan Multiscan 791 CCD camera.

\section{Immunostaining of patient biopsies}

Snap-frozen biopsies of tissue collected from the epicentre of infection from one patient with cellulitis and four with necrotizing fasciitis and toxic shock syndrome caused by M1T1 S. pyogenes isolates were cryosectioned, fixed and immunostained as previously described (Norrby-Teglund et al., 2001) (Thulin et al., 2006). The sections were kindly provided by Prof. Donald E. Low, Mount Sinai Hospital, Toronto, Canada. The study was conducted in accordance with the declaration of Helsinki, and ethical approval to obtain the biopsies was granted by the human subjects review committee at the University of Toronto. Staining for the M1 protein was achieved by incubation with a polyclonal rabbit antiserum against M1 (diluted 1:10 000). Neutrophils were stained with anti-neutrophil elastase (murine IgG1, diluted 1:400, Dako Cytomation), and platelets were stained with anti-CD42a (murine IgG1, diluted 1:200, Serotec). A polyclonal rabbit antiserum against the Lancefield group A carbohydrate was used to detect S. pyogenes (diluted 1:10 000, Difco). Biotinylated secondary antibodies included goat-anti-mouse IgG (diluted 1:300) and goat-anti-rabbit IgG (diluted 1:500, both from Vector Laboratories). Irrelevant isotype-specific murine antibodies (Dako Cytomation) and preimmune rabbit sera at appropriate dilutions were used to control for non-specific staining reactions. The immunostainings were evaluated in a RXM Leica microscope (Leica, Wetzlar, Germany) with a 25X/0.55 NA oil objective lens and 10\% glycerol in PBS as imaging medium. The microscope was equipped with a 3-charge couple device color camera (DXC-750p; Sony Sverige, Spanga, Sweden).

Aggregates of platelets induced by M1 protein were visualized by immunofluorescence stainings carried out using the above mentioned antibodies and streptavidin-conjugated flourophores (Alexa Flour 488 diluted 1:600, Alexa Flour 546 and Alexa Flour 633 both diluted in 1:500; Molecular Probes, Eugene, Oregon, 
United States). All antibodies and fluorochromes were diluted in PBS-saponin-BSAc. M1 protein was visualized using biotinylated polyclonal rabbit anti-M1 IgG antibodies at a concentration of $3 \mu \mathrm{g} / \mathrm{ml}$ and human $\mathrm{IgG}$ were detected by incubation with biotinylated $\mathrm{Ig}$-binding protein $\mathrm{L}$ at a concentration of $0.5 \mu \mathrm{g} / \mathrm{ml}$. Evaluation and visualization was achieved by use of a Leica confocal scanner TCS SP II coupled to a Leica DMR microscope (Leica, Wetzlar, Germany).

\section{Acknowledgments:}

We are indebted to Dr. Heiko Herwald for invaluable advice and discussion of the project. We wish to thank Maria Baumgarten, Anette Hofmann and Ingbritt Gustafsson for excellent technical assistance.

\section{References}

Åkesson, P., Cooney, J., Kishimoto, F., and Björck, L. (1990) Protein H--a novel IgG binding bacterial protein. Mol Immunol 27: 523-531.

Åkesson, P., Schmidt, K.H., Cooney, J., and Björck, L. (1994) M1 protein and protein $\mathrm{H}$ : IgGFc- and albumin-binding streptococcal surface proteins encoded by adjacent genes. Biochem J 300: 877-886.

Andonegui, G., Kerfoot, S.M., McNagny, K., Ebbert, K.V., Patel, K.D., and Kubes, P. (2005) Platelets express functional Toll-like receptor-4. Blood 106: 24172423.

Ashbaugh, C.D., Warren, H.B., Carey, V.J., and Wessels, M.R. (1998) Molecular analysis of the role of the group A streptococcal cysteine protease, hyaluronic acid capsule, and $\mathrm{M}$ protein in a murine model of human invasive soft-tissue infection. J Clin Invest 102: 550-560.

Barker, F.G., Leppard, B.J., and Seal, D.V. (1987) Streptococcal necrotising fasciitis: comparison between histological and clinical features. J Clin Pathol 40: 335341.

Berge, A., and Björck, L. (1995) Streptococcal cysteine proteinase releases biologically active fragments of streptococcal surface proteins. $J$ Biol Chem 270: 9862-9867.

Björck, L. (1988) Protein L. A novel bacterial cell wall protein with affinity for Ig L chains. J Immunol 140: 1194-1197.

Bryant, A.E., Bayer, C.R., Chen, R.Y., Guth, P.H., Wallace, R.J., and Stevens, D.L. (2005) Vascular dysfunction and ischemic destruction of tissue in Streptococcus pyogenes infection: the role of streptolysin O-induced platelet/neutrophil complexes. J Infect Dis 192: 1014-1022.

Carapetis, J.R., Steer, A.C., Mulholland, E.K., and Weber, M. (2005) The global burden of group A streptococcal diseases. Lancet Infect Dis 5: 685-694.

Coller, B.S. (1995) The role of platelets in arterial thrombosis and the rationale for blockade of platelet GPIIb/IIIa receptors as antithrombotic therapy. Eur Heart J 16 Suppl L: 11-15. 
Collin, M., and Olsen, A. (2000) Generation of a mature streptococcal cysteine proteinase is dependent on cell wall-anchored M1 protein. Mol Microbiol 36: 1306-1318.

Cunningham, M.W. (2000) Pathogenesis of group A streptococcal infections. Clin Microbiol Rev 13: 470-511.

de Gaetano, G., Cerletti, C., and Evangelista, V. (1999) Recent advances in plateletpolymorphonuclear leukocyte interaction. Haemostasis 29: 41-49.

Fischetti, V.A. (1989) Streptococcal M protein: molecular design and biological behavior. Clin Microbiol Rev 2: 285-314.

Fitzgerald, J.R., Foster, T.J., and Cox, D. (2006a) The interaction of bacterial pathogens with platelets. Nat Rev Microbiol 4: 445-457.

Fitzgerald, J.R., Loughman, A., Keane, F., Brennan, M., Knobel, M., Higgins, J., Visai, L., Speziale, P., Cox, D., and Foster, T.J. (2006b) Fibronectin-binding proteins of Staphylococcus aureus mediate activation of human platelets via fibrinogen and fibronectin bridges to integrin GPIIb/IIIa and IgG binding to the FcgammaRIIa receptor. Mol Microbiol 59: 212-230.

Ford, I., Douglas, C.W., Cox, D., Rees, D.G., Heath, J., and Preston, F.E. (1997) The role of immunoglobulin $G$ and fibrinogen in platelet aggregation by Streptococcus sanguis. Br J Haematol 97: 737-746.

Gawaz, M., Dickfeld, T., Bogner, C., Fateh-Moghadam, S., and Neumann, F.J. (1997) Platelet function in septic multiple organ dysfunction syndrome. Intensive Care Med 23: 379-385.

Greenman, J., Tutt, A.L., George, A.J., Pulford, K.A., Stevenson, G.T., and Glennie, M.J. (1991) Characterization of a new monoclonal anti-Fc gamma RII antibody, AT10, and its incorporation into a bispecific $F\left(a b^{\prime}\right) 2$ derivative for recruitment of cytotoxic effectors. Mol Immunol 28: 1243-1254.

Herwald, H., Cramer, H., Morgelin, M., Russell, W., Sollenberg, U., Norrby-Teglund, A., Flodgaard, H., Lindbom, L., and Björck, L. (2004) M protein, a classical bacterial virulence determinant, forms complexes with fibrinogen that induce vascular leakage. Cell 116: 367-379.

Kantor, F.S. (1965) Fibrinogen precipitation by streptococcal M protein. J Exp Med 1;121: 849-859.

Kirschenbaum, L.A., Adler, D., Astiz, M.E., Barua, R.S., Saha, D., and Rackow, E.C. (2002) Mechanisms of platelet-neutrophil interactions and effects on cell filtration in septic shock. Shock 17: 508-512.

Levi, M., de Jonge, E., and van der Poll, T. (2003) Sepsis and disseminated intravascular coagulation. J Thromb Thrombolysis 16: 43-47.

Li, N., Goodall, A.H., and Hjemdahl, P. (1997) A sensitive flow cytometric assay for circulating platelet-leucocyte aggregates. Br J Haematol 99: 808-816.

Li, N., Hu, H., Lindqvist, M., Wikstrom-Jonsson, E., Goodall, A.H., and Hjemdahl, P. (2000) Platelet-leukocyte cross talk in whole blood. Arterioscler Thromb Vasc Biol 20: 2702-2708.

Loughman, A., Fitzgerald, J.R., Brennan, M.P., Higgins, J., Downer, R., Cox, D., and Foster, T.J. (2005) Roles for fibrinogen, immunoglobulin and complement in platelet activation promoted by Staphylococcus aureus clumping factor A. Mol Microbiol 57: 804-818.

Mavrommatis, A.C., Theodoridis, T., Orfanidou, A., Roussos, C., ChristopoulouKokkinou, V., and Zakynthinos, S. (2000) Coagulation system and platelets are fully activated in uncomplicated sepsis. Crit Care Med 28: 451-457. 
Norrby-Teglund, A., Thulin, P., Gan, B.S., Kotb, M., McGeer, A., Andersson, J., and Low, D.E. (2001) Evidence for superantigen involvement in severe group a streptococcal tissue infections. J Infect Dis 184: 853-860.

Påhlman, L.I., Mörgelin, M., Eckert, J., Johansson, L., Russell, W., Riesbeck, K., Soehnlein, O., Lindbom, L., Norrby-Teglund, A., Schumann, R.R., Björck, L., and Herwald, H. (2006) Streptococcal M protein: a multipotent and powerful inducer of inflammation. J Immunol 177: 1221-1228.

Russwurm, S., Vickers, J., Meier-Hellmann, A., Spangenberg, P., Bredle, D., Reinhart, K., and Losche, W. (2002) Platelet and leukocyte activation correlate with the severity of septic organ dysfunction. Shock 17: 263-268.

Sjöbring, U., Ringdahl, U., and Ruggeri, Z.M. (2002) Induction of platelet thrombi by bacteria and antibodies. Blood 100: 4470-4477.

Stahl, A.L., Svensson, M., Morgelin, M., Svanborg, C., Tarr, P.I., Mooney, J.C., Watkins, S.L., Johnson, R., and Karpman, D. (2006) Lipopolysaccharide from enterohemorrhagic Escherichia coli binds to platelets through TLR4 and CD62 and is detected on circulating platelets in patients with hemolytic uremic syndrome. Blood 108: 167-176.

Stevens, D.L. (2001) Invasive streptococcal infections. J Infect Chemother 7: 69-80.

Thulin, P., Johansson, L., Low, D.E., Gan, B.S., Kotb, M., McGeer, A., and NorrbyTeglund, A. (2006) Viable Group A Streptococci in Macrophages during Acute Soft Tissue Infection. PLoS Med 3: e53.

von Pawel-Rammingen, U., Johansson, B.P., and Björck, L. (2002) IdeS, a novel streptococcal cysteine proteinase with unique specificity for immunoglobulin G. EMBO J 21: 1607-1615.

Wachowicz, B., Saluk, J., and Kaca, W. (1998) Response of blood platelets to Proteus mirabilis lipopolysaccharide. Microbiol Immunol 42: 47-49.

Weyrich, A.S., Lindemann, S., and Zimmerman, G.A. (2003) The evolving role of platelets in inflammation. J Thromb Haemost 1: 1897-1905.

Zimmerman, G.A., McIntyre, T.M., Prescott, S.M., and Stafforini, D.M. (2002) The platelet-activating factor signaling system and its regulators in syndromes of inflammation and thrombosis. Crit Care Med 30: S294-301. 
Table 1: Activation of platelets in plasma as determined using flow cytometry after treatment with $S$. pyogenes bacteria, M1 protein, Protein $H, M 1$ protein fragment A-S, and M1 protein fragment S-C3.

\begin{tabular}{|l|ccc|}
\hline \multirow{2}{*}{ Agonist } & \multicolumn{3}{|c|}{ Platelet activation marker ${ }^{\text {a }}$} \\
\hline Buffer & PAC-1 & CD62 & Fibrinogen \\
ADP $(5 \mu \mathrm{M})$ & $53 \pm 6 \%$ & $7 \pm 1 \%$ & $5 \pm 1 \%$ \\
S. pyogenes AP1 & $71 \pm 12 \%$ & $67 \pm 8 \%$ & $63 \pm 7 \%$ \\
$\left(1 \times 10^{8} / \mathrm{ml}\right)$ & & & $55 \pm 7 \%$ \\
$\mathrm{M} 1 \mathrm{protein}$ & $73 \pm 5 \%$ & $64 \pm 11 \%$ & $70 \pm 5 \%$ \\
$(1 \mu \mathrm{g} / \mathrm{ml})$ & $3 \pm 1 \%$ & $2 \pm 1 \%$ & $4 \pm 1 \%$ \\
Protein $\mathrm{H}$ & & & $48 \pm 3 \%$ \\
$(10 \mu \mathrm{g} / \mathrm{ml})$ & $64 \pm 9 \%$ & $69 \pm 9 \%$ & $13 \pm 2 \%$ \\
$\mathrm{M} 1 \mathrm{protein}(\mathrm{A}-\mathrm{S})$ & & $10 \pm 6 \%$ & \\
$(10 \mu \mathrm{g} / \mathrm{ml})$ & $8 \pm 4 \%$ & & \\
$\mathrm{M} 1 \mathrm{protein}(\mathrm{S}-\mathrm{C} 3)$ & & & \\
$(10 \mu \mathrm{g} / \mathrm{ml})$ & & & \\
\hline
\end{tabular}

a. Data are expressed as the percentage positive cells for each marker \pm standard error, $n=5$. 
Table 2: Activation of neutrophils and monocytes in whole blood treated with M1 protein $(1 \mu \mathrm{g} / \mathrm{ml})$ or buffer alone, as determined using flow cytometry.

\begin{tabular}{|l|cc|cc|}
\hline \multirow{2}{*}{ Cell surface marker ${ }^{a}$} & \multicolumn{2}{|c|}{ Neutrophils } & \multicolumn{2}{c|}{ Monocytes } \\
& Buffer & M1 protein & Buffer & M1 protein \\
\hline CD42 & $5 \pm 1 \%$ & $52 \pm 4 \%$ & $6 \pm 3 \%$ & $74 \pm 5 \%$ \\
CD11b & $4 \pm 1 \%$ & $64 \pm 6 \%$ & $4 \pm 3 \%$ & $58 \pm 5 \%$ \\
CD66b & $3 \pm 1 \%$ & $52 \pm 8 \%$ & $3 \pm 1 \%$ & $3 \pm 1 \%$ \\
Tissue factor & $2 \pm 3 \%$ & $12 \pm 1 \%$ & $2 \pm 1 \%$ & $45 \pm 3 \%$ \\
\hline
\end{tabular}

${ }^{\text {a. }}$ Data are expressed as the percentage positive cells for each marker \pm standard error, $n=3$. 


\section{Figure Legends}

Figure 1: Microthrombi in $S$. pyogenes infected tissue contain aggregated platelets. The presence of platelets at the local site of infection was assessed in tissue biopsies from patients with soft tissue infection caused by $S$. pyogenes. Biopsy sections were immunohistochemically stained using CD42 as a platelet specific marker. Shown is an area of the tissue at a 40x/0.55 NA oil objective, where platelets appear as positively stained brown aggregates.

Figure 2: Platelet activation by $M 1$ protein requires fibrinogen and IgG. A. Platelet activation was determined using flow cytometry for the binding of PAC-1 antibody. Results are expressed as the percentage of the platelet population that are positive for PAC-1.FITC, after treatment with ADP $(5 \mu \mathrm{M})$ or M1 protein $(1 \mu \mathrm{g} / \mathrm{ml})$. Incubations were carried out in platelet rich plasma (PRP) that was treated with the agonist alone (dark grey bars), or the agonist after preincubation with ReoPro to abolish platelet fibrinogen binding (black bars), AT10 to abolish platelet Fc receptor binding (white bars) or IdeS to cleave plasma IgG (light grey bars). Data are expressed as mean \pm standard error, $\mathrm{n}=5$. $\mathrm{P}$ values were determined using the Students $\mathrm{t}$ test, $\mathrm{p}<0.001 * * *, \mathrm{p}<0.01 * *$.

Figure 3: M1 protein binds to platelets via fibrinogen but binding of IgG is required for platelet activation. A. Binding of M1.FITC to platelets and activation status of the platelets was determined using flow cytometry. Results are expressed as the percentage of the platelet population that are positive for M1.FITC (dark grey bars) or CD62.PE (white bars), after treatment with M1.FITC $(2 \mu \mathrm{g} / \mathrm{ml})$. Incubations were carried out in PRP that was untreated, or that had been pre-incubated with ReoPro or AT10. Data are expressed as mean \pm standard error, $n=5$. P values were determined using the Students t test, $\mathrm{p}<0.001 * * *, \mathrm{p}<0.01 * *$. B. The binding of M1.FITC to platelets and the activation status of the platelets was determined as the percentage of the platelet population that were positive for M1.FITC, CD62.PE or PAC-1.FITC, after treatment with ADP $(5 \mu \mathrm{M})$ or M1.FITC $(2 \mu \mathrm{g} / \mathrm{ml})$. Incubations were carried out in PRP that was untreated (dark grey bars), or in PRP that had been pre-incubated with a cocktail of platelet inhibitors (white bars). Data are expressed as mean \pm standard error, $n=3$.

Figure 4: IgG dependent variation in platelet activation by M1 protein in different donors A. ELISA was used to determine IgG titres against M1 protein in the sera of five different donors (unbroken lines) and in the purified anti-M1 protein IgG preparation (broken line). B. Platelet activation was determined using flow cytometry for surface CD62.PE in PRP from the same five donors as shown in A (unbroken lines) and one non responsive donor supplemented with purified anti-M1 protein IgG (broken line). Results are expressed as the percentage of the platelet population that are positive for CD62.PE, after treatment with M1 (0 to $4 \mu \mathrm{g} / \mathrm{ml}$ )

Figure 5: M1 protein induces the formation of platelet-leucocyte aggregates. The generation of platelet-leucocyte aggregates was determined using flow cytometry. Results are expressed as the percentage of the neutrophil or monocyte population that are positive for the platelet specific marker, CD42, after treatement with M1 protein ( $2 \mu \mathrm{g} / \mathrm{ml}$ ). Incubations were carried out in whole blood from different donors that 
were treated with M1 protein alone (white bars) or M1 protein after preincubation of whole blood with ReoPro to abolish platelet fibrinogen binding (black bars), or AT10 to abolish platelet $\mathrm{Fc}$ receptor binding (light grey bars). Data are expressed as mean \pm standard error, $\mathrm{n}=5$. $\mathrm{P}$ values were determined using the Students $\mathrm{t}$ test, $\mathrm{p}<0.001 * * *$, $\mathrm{p}<0.01 * *$.

Figure 6: M1 protein mediates the formation of platelet rich thrombi. Scanning electron microscopy was used to determine the morphology of platelets after 1 minute incubation with buffer (A), $5 \mu \mathrm{M}$ ADP (B), or $1 \mu \mathrm{g} / \mathrm{ml} \mathrm{M} 1$ protein (C). The length of the bar corresponds to $20 \mu \mathrm{m}$.

Figure 7: S. pyogenes infected tissue contains aggregates of platelets, M1 protein and immunoglobulins. The presence of platelets, neutrophils, S. pyogenes and the M1 protein at the local site of infection was assessed by immunostainings of tissue biopsies from patients with soft tissue infection caused by M1 isolates, as described in Experimental procedures. The figure shows results from one representative patient, and similar results were seen in biopsies from four additional patients. (A) Consecutive biopsy sections were immunohistochemically stained for different markers as indicated in the figure. Positive stainings are shown in brown and cellular infiltrates by hematoxylin counterstain (blue). Shown is the same area of the tissue at a $25 \times / 0.55$ NA oil objective (upper panel). A higher magnification of the area indicated by the white rectangle is shown (lower panel). (B) Aggregates of platelets (Alexa 633, magenta) and $S$. pyogenes (Alexa 488, green) in the tissue biopsies was visualised by immunoflourescent stainings and analysed by confocal microscopy. Cellular infiltrates are indicated in blue by the DNA binding stain DAPI. Bar: $10 \mu \mathrm{m}$ (C) Aggregates of platelets (Alexa 633, magenta), IgG (Alexa 488, green) and M1 protein (Alexa 546, yellow) were visualized as described in (B) and are indicated by white arrows. Bar: $20 \mu \mathrm{m}$. 
Figure 1

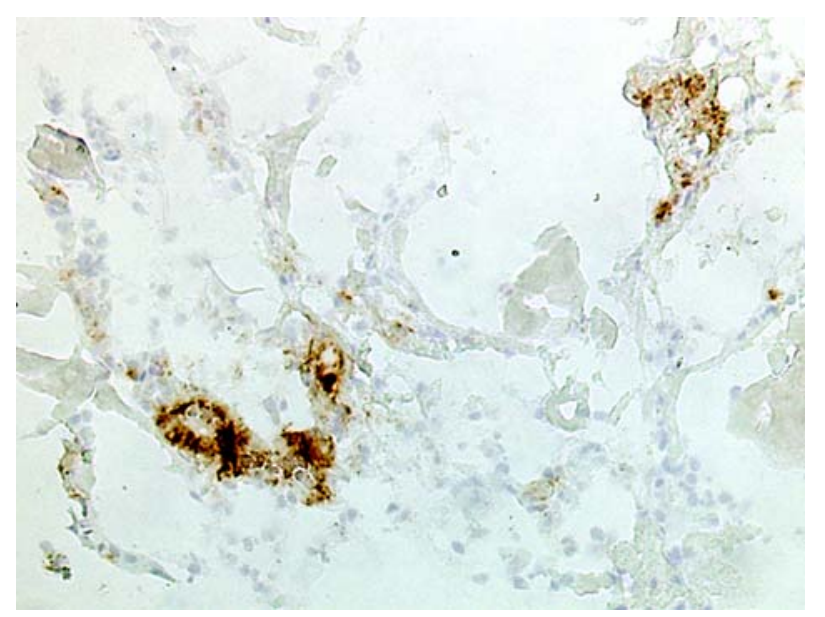

Figure 2

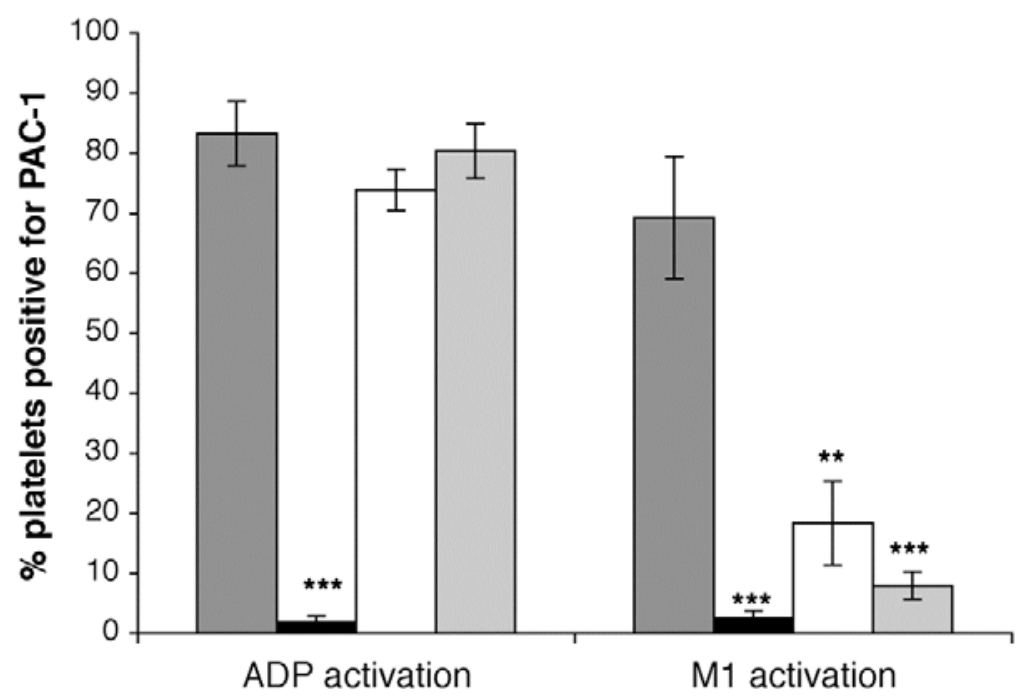


Figure 3

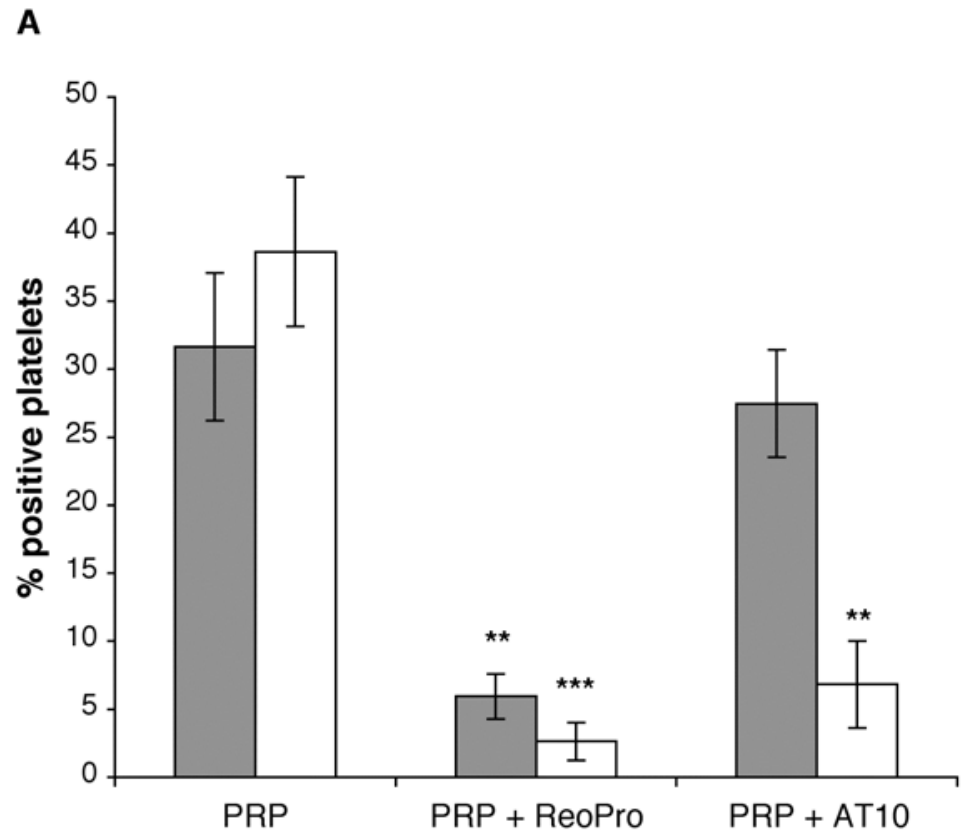

B

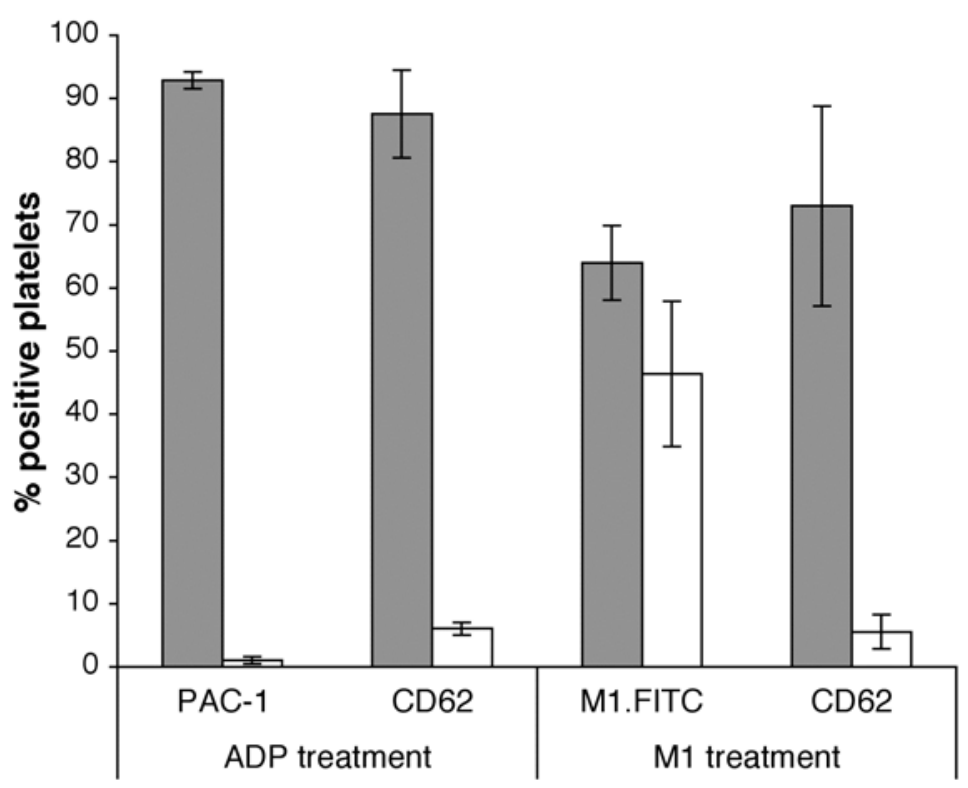


Figure 4

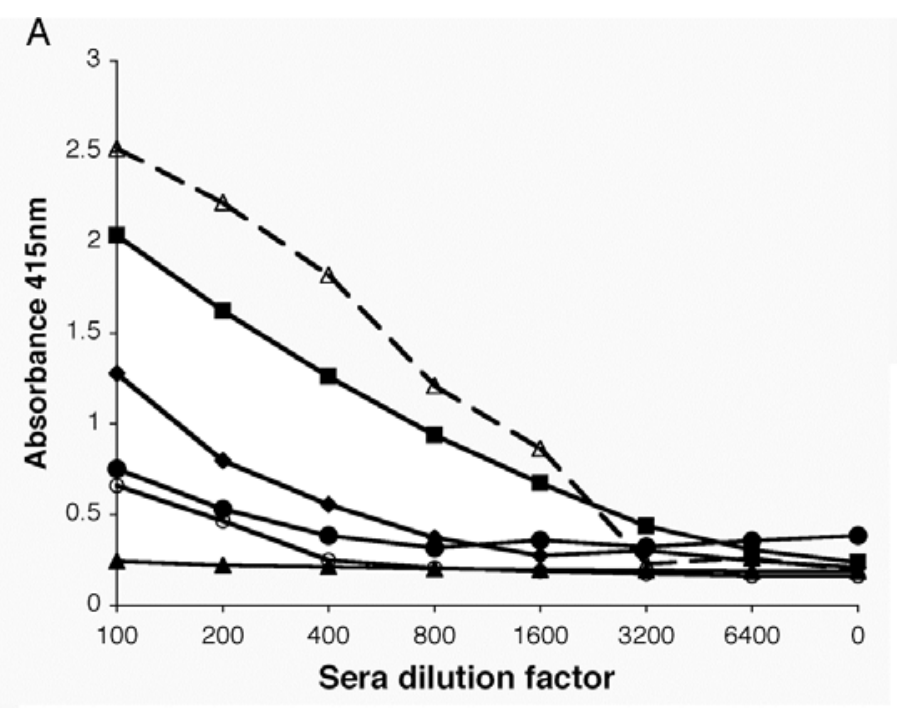

B

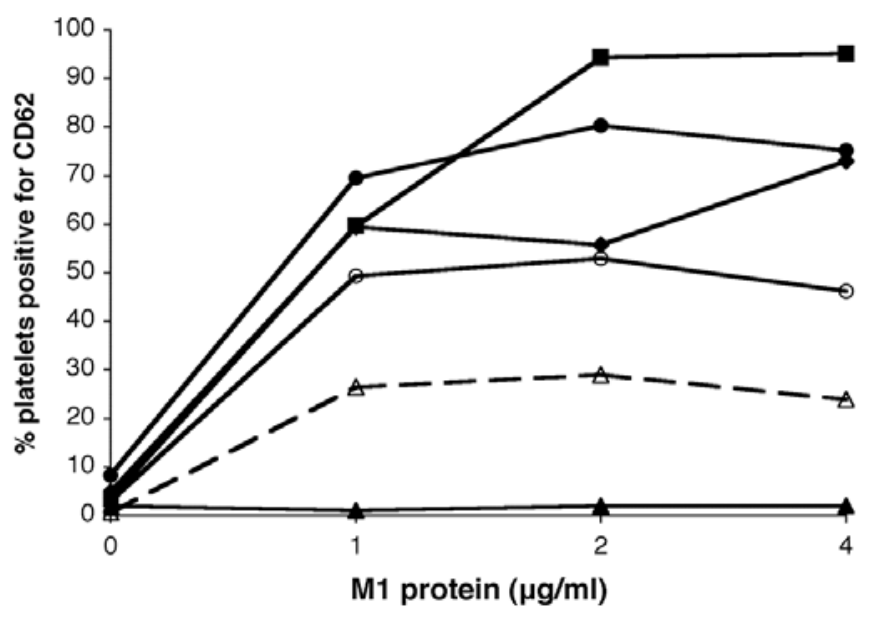


Figure 5

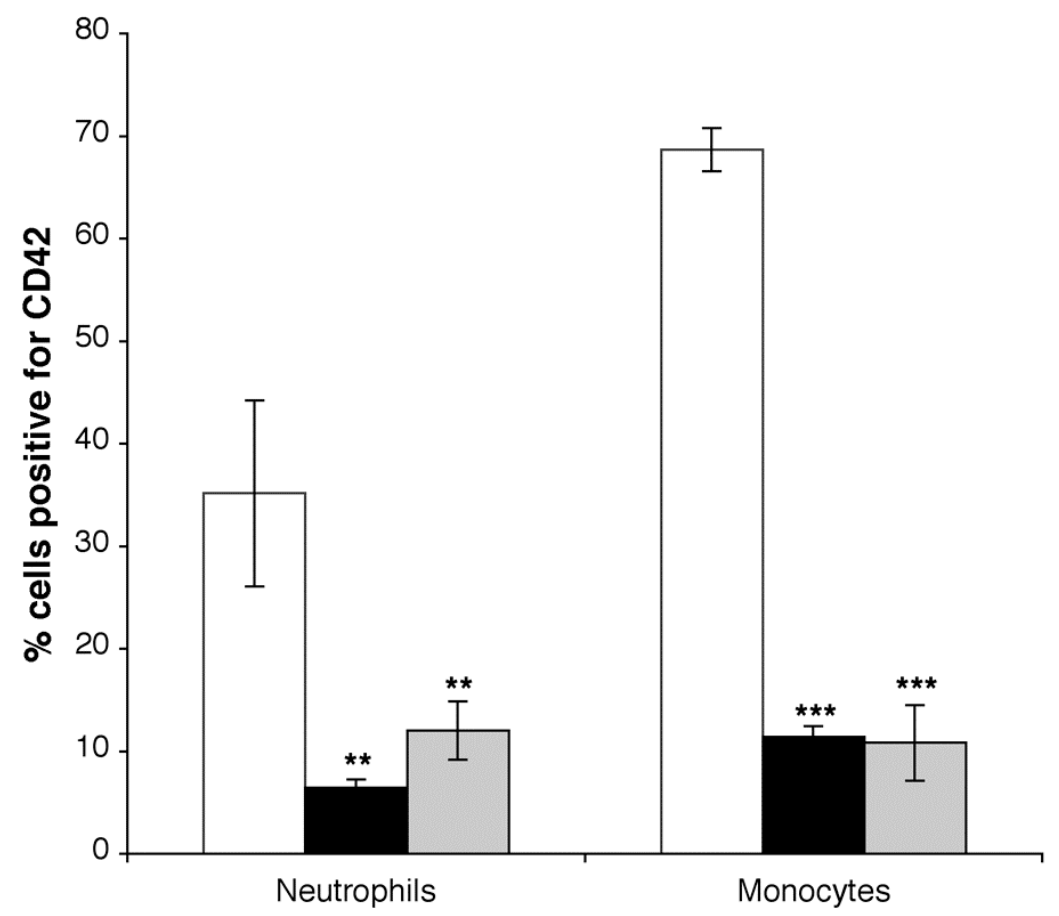


Figure 6
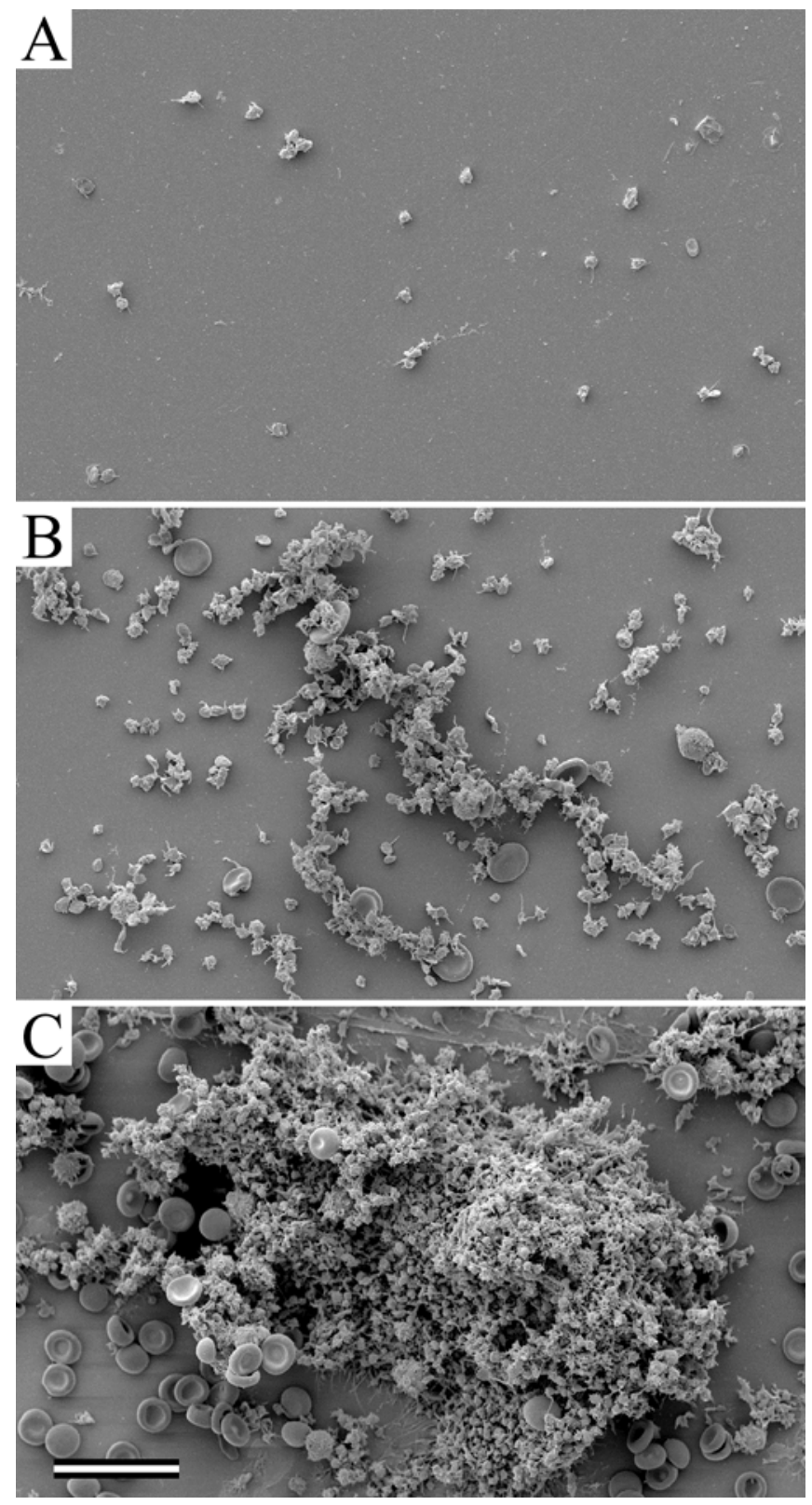
Figure 7

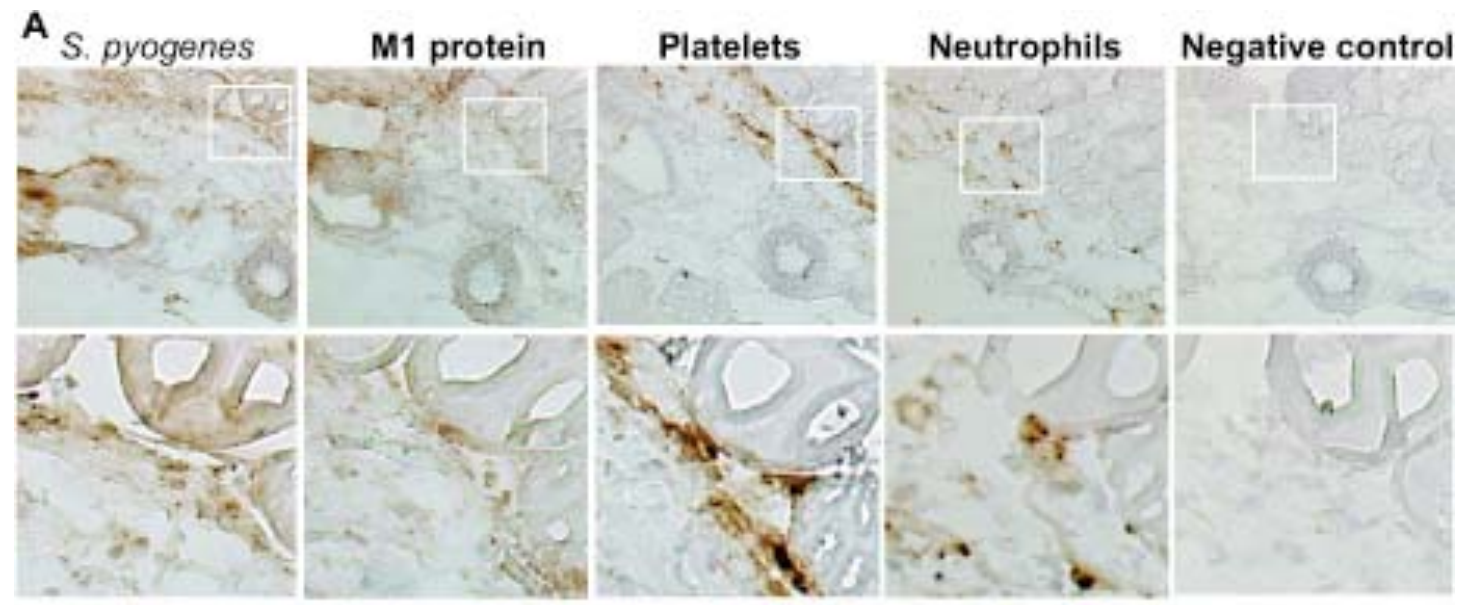

B
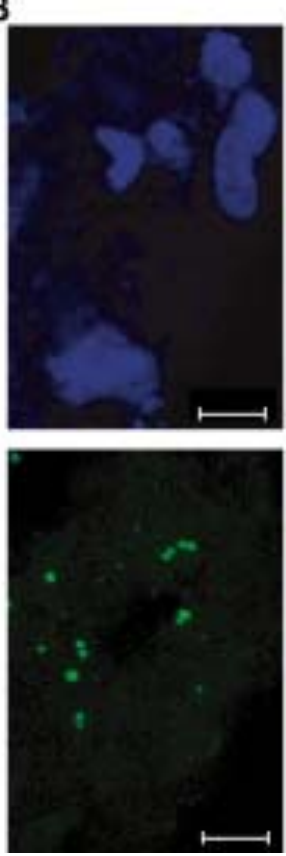
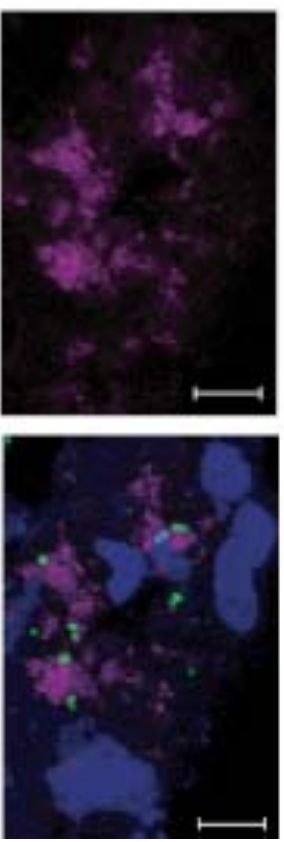

C
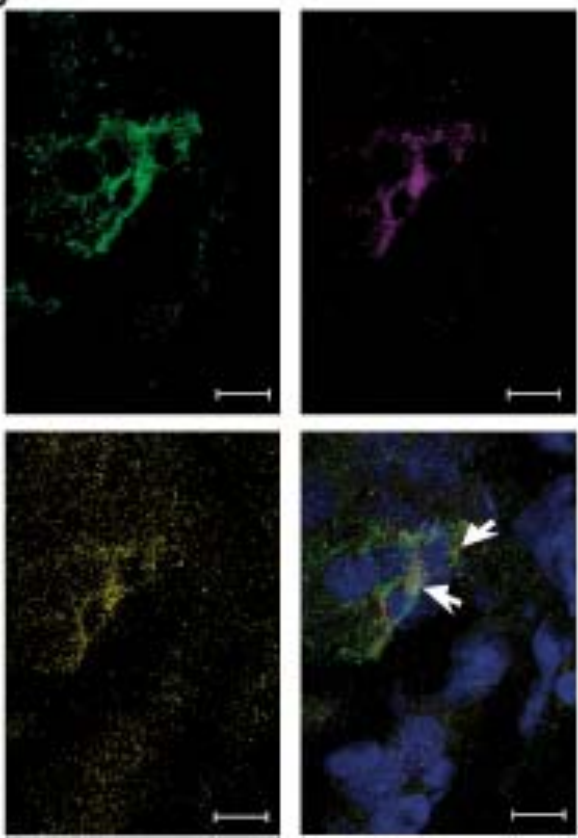